EXTENDED REPORT

\title{
Incidence of keratitis of varying severity among contact lens wearers
}

\author{
P B Morgan, N Efron, E A Hill, M K Raynor, M A Whiting, A B Tullo
}

Br J Ophthalmol 2005;89:430-436. doi: 10.1136/bjo.2004.052688

See end of article for authors' affiliations .....................

Correspondence to: Philip B Morgan, Department of Optometry, The University of Manchester, PO Box 88, Manchester M60 1QD UK; philip.morgan@ manchester.ac.uk

Accepted for publication 29 August 2004

\begin{abstract}
Aim: To determine the incidence of non-severe keratitis (NSK) and severe keratitis (SK) among wearers of current generation contact lenses.

Methods: A 12 month, prospective, hospital based epidemiological study was conducted by examining all contact lens wearers presenting with a corneal infiltrate/ulcer to a hospital centre in Manchester. A clinical severity matrix was used to differentiate between NSK and SK, based on the severity of signs and symptoms. The size of the hospital catchment population and the wearing modalities (daily wear (DW) or extended wear (EW)) and lens types being used were estimated from relevant demographic and market data.

Results: During the survey period, 80 and 38 patients presented with NSK and SK, respectively. The annual incidences (cases per 10000 wearers) for each wearing modality and lens type were: DW rigidNSK 5.7, SK 2.9; DW hydrogel daily disposable-NSK 9.1, SK 4.9; DW hydrogel (excluding daily disposable)-NSK 14.1, SK 6.4; DW silicone hydrogel-NSK 55.9, SK 0.0; EW rigid-NSK 0.0, SK 0.0; EW hydrogel-NSK 48.2, SK 96.4; EW silicone hydrogel-NSK 98.8, SK 19.8. The difference in SK between EW hydrogel and EW silicone hydrogel was significant $(p=0.04)$.

Conclusions: A clinical severity matrix has considerable utility in assessing contact lens related keratitis. There is a significantly higher incidence of SK in wearers who sleep in contact lenses compared with those who only use lenses during the waking hours. Those who choose to sleep in lenses should be advised to wear silicone hydrogel lenses, which carry a five times decreased risk of SK for extended wear compared with hydrogel lenses.
\end{abstract}

$\mathrm{T}$ he landscape of contact lens practice has changed dramatically over the past decade, with the introduction of new contact lens genres such as daily disposable lenses $^{12}$ and silicone hydrogel materials. ${ }^{3}$ It might be expected that these new products will be associated with lower levels of ocular morbidity, as well as providing greater levels of comfort and convenience for contact lens wearers. For example, daily disposable lenses could reduce the risk of ocular complications ${ }^{12}$ because they obviate the need for lens maintenance and avoid problems related to long term surface spoilation. Silicone hydrogel lenses should minimise clinical problems relating to corneal hypoxia because of their greater capacity to transmit atmospheric oxygen to the ocular surface ${ }^{4}$ Despite these significant advances, there have been recent case reports of severe microbial keratitis with daily disposable lenses ${ }^{5}$ and silicone hydrogel lenses. ${ }^{67}$

The aim of this study was to undertake a prospective epidemiological investigation of the safety of all current forms of contact lens wear. We employed a previously described novel methodological paradigm capable of differentiating the severity of keratitis associated with contact lens wear.

\section{METHODS}

\section{Study setting}

A prospective survey was conducted between 25 January 2003 and 24 January 2004 of all patients, who were wearing contact lenses (whether or not this was the primary reason for presentation), attending the acute service of the Royal Eye Hospital, Manchester, United Kingdom (the study centre).

This research followed the tenets of the Declaration of Helsinki. Informed consent was obtained from all patients surveyed in this study. Ethical approval to conduct this study was obtained from the local research ethics committee (central) of Manchester Health Authority.

\section{Survey procedure}

During the survey period, all presenting patients were asked if they habitually wore contact lenses. Patients giving an affirmative response were asked to complete a green coloured patient survey questionnaire (fig l) to provide information such as personal details, whether or not they sleep in lenses (wearing modality), lens type (defined in terms of the material from which the lens is fabricated and/or the lens replacement frequency), lens care system used, and their reason for attending the study centre.

When a patient was examined, the presence of any form of corneal infiltrate or ulcer was noted. If there were no evidence of such corneal involvement, no further information was captured for the purposes of this survey. If there were a corneal infiltrate or ulcer, the attending clinician completed a red coloured clinical survey form (on the reverse side of the patient survey questionnaire; fig 1) which gathered clinical information relevant to this study. The colour coding of the survey forms facilitated ease of implementation in a busy clinical environment.

A key feature of the clinical data captured was the use of a clinical severity matrix as described by Aasuri et $a l^{8}$ (with minor textual adaptations) (fig 1). This involved the clinician encircling a severity descriptor (on a 0 to 3 scale) with respect to 10 pertinent signs and symptoms, providing a mechanism for classifying corneal infiltrative events as non-severe or severe. The cumulative score for each event-the "clinical severity score" -was between 2 (the minimum score relating to the criterion for this study of only including patients exhibiting an infiltrative response) and 22, whereby the higher the score, the more clinically severe the event. Aasuri

Abbreviations: DW, daily wear; EW, extended wear; NSK, non-severe keratitis; PMMA, poly(methyl methacrylate); SK, severe keratitis 


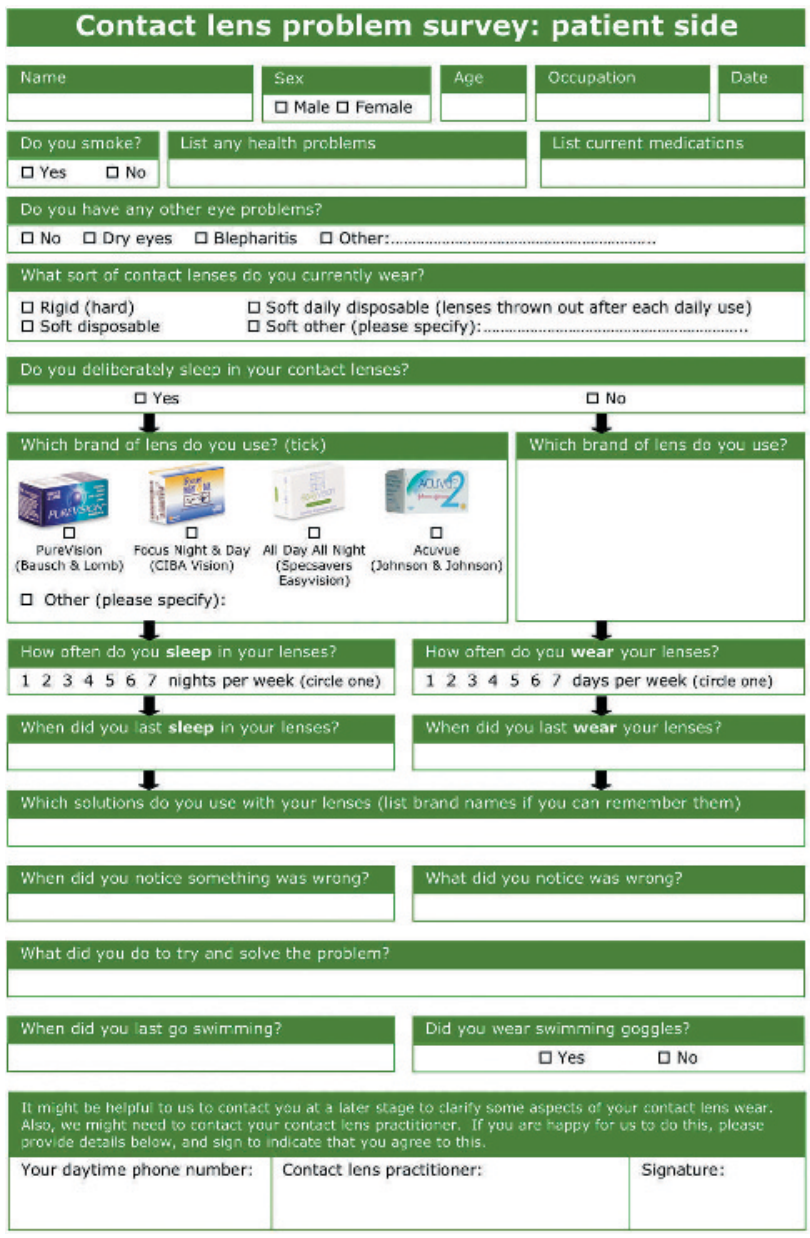

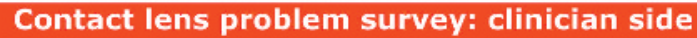

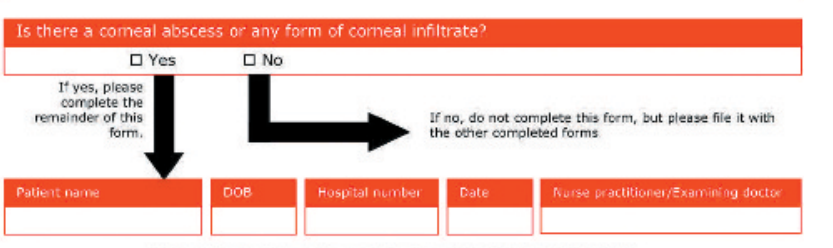

Orcle one descriptor on each row, for the parameters listed in the table below.

\begin{tabular}{|c|c|c|c|c|}
\hline Parameter & 0 & 1 & 2 & 3 \\
\hline symptoms & Nore & Mild & Mederate & Severe \\
\hline Lid swelling & Absent & & Present & \\
\hline Conjunctival redness & Absent & Lecalised & Generalised & \\
\hline Infiltrate s7ape ${ }^{x}$ & & Found & & Irregular \\
\hline Infiltrate sze (brgest dismeter) & & $51.0 \mathrm{~mm}$ & $1.0-2.0 \mathrm{~mm}$ & $22.0 \mathrm{~mm}$ \\
\hline Fuorescein staining & Aosent & Present & & \\
\hline Surrourding comes & Clear & Sllght haze & Severe haze & \\
\hline Endothelial debris & Absent & Present & & \\
\hline Hypopysn & Absent & & Present & \\
\hline Effect of lens discontinuation*x & Resolving & No charge & Slight worsening & Significant worsening \\
\hline
\end{tabular}

$\mathrm{R}$
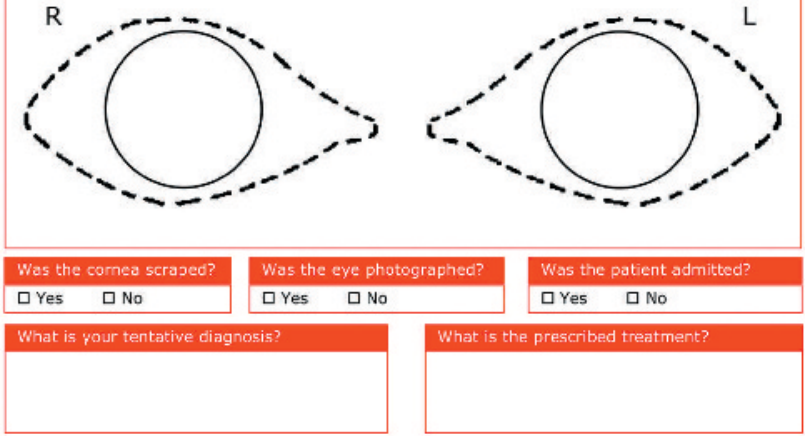

Figure 1 Survey forms completed by patient (green) and clinician (red). The clinical severity matrix referred to in the text can be seen in the middle of the clinician survey form.

et $a l^{8}$ have validated this scoring system indicating that any score greater than 8 should raise a high index of suspicion of clinically severe microbial keratitis.

This study required the attending clinician to indicate if a corneal scrape for culture was performed, a decision based on their own clinical judgment and unrelated to the conduct of this study. The results of any such scrapes were recorded. Only information relating to wearing modality, lens type, clinical severity score, and outcome of any corneal scrapes undertaken was analysed. Additional information gathered during this survey relating to potential risk factors and outcomes will be the subject of further analysis and is not reported here.

\section{Terminology}

In this paper, we use the term "severe keratitis" to describe any clinical presentation which attracted a clinical severity score of more than 8; in conventional clinical parlance, this is presumed to be a case of severe microbial keratitis (essentially the designation given by Aasuri et $a l^{8}$ ) which requires intensive antimicrobial treatment.

The term "non-severe keratitis"" is used to describe any clinical presentation which attracted a clinical severity score of 8 or less; conditions with this range of scores do not typically require medical intervention. We have chosen to use this term rather than the term "sterile keratitis" adopted by Aasuri $e t{ }^{8} l^{8}$ in view of uncertainty relating to the aetiology and pathogenesis of this category of events. ${ }^{10}$

\section{Patient follow up}

To provide assurance of the quality of data collected, an attempt was made to telephone all patients and/or their contact lens practitioner (with permission from the patient). Any unclear, ambiguous, or incomplete data entries were clarified and any required amendments were recorded.

\section{Hospital catchment population}

Calculation of the incidence of infiltrative events necessitated an estimation of the catchment population of the study centre. We applied the technique of Bailey (reported by Senn and Sampson ${ }^{11}$ ) to demographic details of the 24630 patients with all forms of ocular problems presenting to the study centre during the survey period. Essentially, this involved determining in which of the 14 National Health Service primary care trusts (urban geographic regions) within Greater Manchester the patients were resident. The small number of patients resident outside Greater Manchester was also accounted for in this analysis. Assuming that the rates of ocular problems were similar for all areas of residence, a weighted population size was calculated for each area and the sum total of these was determined to be 1071 503-the "hospital catchment population."

\section{Determination of lens wearing modality}

We have conducted an annual survey on contact lens prescribing trends in the United Kingdom for the past 9 years. ${ }^{12}$ This data set comprises information relating to 
8833 contact lens fits by 977 practitioners. From these data, we could determine the proportion of each of the four lens types described below that are prescribed for daily wear versus extended wear.

\section{Categorisation of lens type}

We categorised contact lens types as follows:

- Rigid-lenses made from silicone-containing rigid gas permeable materials. (Virtually no wearers have been fitted with poly(methyl methacrylate) (PMMA) lenses over the past 9 years in the United Kingdom. ${ }^{12}$ )

- Hydrogel daily disposable-single use hydrogel lenses (excluding silicone hydrogel lenses) which are discarded after each daily use

- Hydrogel-all forms of hydrogel lenses (excluding daily disposable and silicone hydrogel lenses). Over the past 9 years, $4 \%$ of wearers of this lens type have been prescribed lenses to be replaced fortnightly, $70 \%$ monthly, and $18 \%$ less frequently; $8 \%$ of wearers were advised to replace lenses as and when required (that is, on an unplanned replacement basis) ${ }^{12}$

- Silicone hydrogel-new generation silicone-containing hydrogel lenses; during the time of this survey, only two such materials were available in the United Kingdom (balafilcon A and lotrafilcon A). Both of these lens types are prescribed for monthly replacement. ${ }^{12}$

\section{Determination of number of lens wearers}

Information on the number of contact lens wearers in the United Kingdom in each of the above lens type categories was accessed from lens sales data collected by the UK Association of Contact Lens Manufacturers for 2003. ${ }^{13}$ Using the data outlined above on wearing modality and lens type, and assuming a total UK population of $59050800,{ }^{14}$ we were able to estimate the number of wearers within the hospital catchment population using each lens type on a daily wear versus extended wear basis. The above analyses assume that lens use in the hospital catchment population is the same as that for the United Kingdom as a whole.

\section{Data analysis}

For each wearing modality/lens type combination investigated in this 12 month study, the annual incidence of nonsevere and severe keratitis was determined by dividing the number of cases of each of these conditions by the estimated number of wearers for each wearing modality/lens type combination within the hospital catchment population. In common with previous practice in this field, we have expressed incidence data as the number of cases per 10000 wearers per year. The $95 \%$ confidence intervals (CI) for all incidence estimates were calculated according to the methodology of Wilson. ${ }^{15}$ The data are also expressed in terms of "relative risk," which is the ratio of annual incidence of a given condition to an arbitrary "referent" condition, ${ }^{16}$ which we have chosen to be daily wear hydrogel lenses.
The significance of differences between various combinations of annual incidence was determined using the $\chi^{2}$ test. For comparisons involving small numbers of presenting patients (that is, less than five for any cell within the analysis), the Fisher's exact test variant of the $\chi^{2}$ test was employed.

\section{RESULTS}

\section{Wearing modality and lens type}

The number of wearers of each combination of wearing modality/lens type in the United Kingdom and the hospital catchment population is presented in table 1 .

\section{Survey outcome}

Over the 12 month survey period, 415 patients who were wearing contact lenses presented to the study centre. None of these patients was wearing PMMA lenses. All of these patients except one agreed to complete a survey form. Five forms were unintelligible and therefore were not analysed in this study. The remaining usable forms provided details of 118 patients who were observed to have a corneal infiltrate or ulcer. For these patients, a clinical survey form was completed by the attending clinician while examining the patient. A clinical severity score for each case was subsequently determined from the sum of the gradings on the clinical severity matrix.

Figure 2 shows the distribution of clinical severity scores for the 118 patients included in this analysis, stratified by wearing modality and lens type. According to the scoring system, 80 patients presented with non-severe keratitis and 38 with severe keratitis. The number of patients who presented with non-severe and severe keratitis with respect to each of the two wearing modalities and four lens types is presented in table 2 .

Table 2 documents one case of non-severe keratitis and two cases of severe keratitis in patients wearing hydrogel daily disposable lenses on an "extended wear" basis. Although such lenses are self evidently prescribed for daily wear, in these three cases the patients admitted to being non-compliant by way of sleeping in lenses immediately

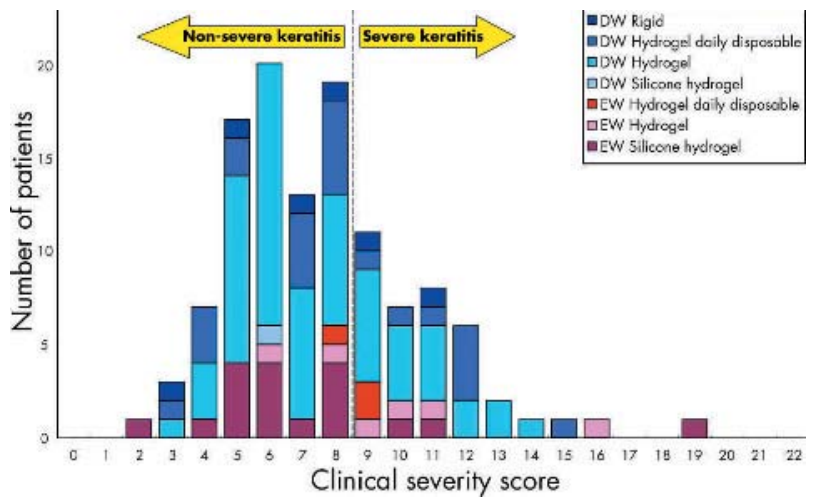

Figure 2 Distribution of clinical severity scores for non-severe and severe keratitis stratified by wearing modality and lens type.

Table 1 Contact lens demographics

\begin{tabular}{lccllrr}
\hline Lens type & Wearers in UK & Wearers in HCP* & Daily wear & Extended wear & Daily wearers in HCP & Extended wearers in HCP \\
\hline Rigid & 386186 & 7014 & $99.75 \%$ & $0.25 \%$ & 6996 & 18 \\
Hydrogel daily disposable & 903765 & 16413 & $100.00 \%$ & $0.00 \%$ & 16413 & 0 \\
Hydrogel & 1667905 & 30291 & $98.63 \%$ & $1.37 \%$ & 29876 & 179 \\
Silicone hydrogel & 93410 & 1696 & $10.55 \%$ & $89.45 \%$ & & 1517 \\
\hline
\end{tabular}




\begin{tabular}{|c|c|c|c|c|}
\hline Wearing modality & Lens type & Non-severe keratitis & Severe keratitis & Total \\
\hline \multirow[t]{4}{*}{ Daily wear } & Rigid & 4 & 2 & 6 \\
\hline & Hydrogel daily disposable & 15 & 8 & 23 \\
\hline & Hydrogel & 42 & 19 & 61 \\
\hline & Silicone hydrogel & 1 & 0 & 1 \\
\hline \multirow[t]{4}{*}{ Extended wear } & Rigid & 0 & 0 & 0 \\
\hline & Hydrogel daily disposable & 1 & 2 & 3 \\
\hline & Hydrogel & 2 & 4 & 6 \\
\hline & Silicone hydrogel & 15 & 3 & 18 \\
\hline Total & & 80 & 38 & 118 \\
\hline
\end{tabular}

before presentation at the hospital; data relating to these cases are therefore entered as extended wear incidents.

\section{Patient follow up}

Our quality assurance procedure resulted in 70 of these 118 patients and/or their contact lens practitioners being contacted directly. Of this subgroup, data relating to 67 patients were determined to be accurate and adjustments were needed with respect to the other three patients. For the 48 patients/practitioners who could not be contacted-or declined permission to be contacted-it was judged that the information supplied was sufficiently clear to be included in the analysis.

\section{Incidence and relative risk of non-severe and severe keratitis}

Incidence and relative risk data are presented in table 3. Comparisons of the statistical significance of differences in incidence of non-severe and severe keratitis between various pairs of combinations of wearing modality/lens type are displayed in table 4. Although caution must be exercised in interpreting $\mathrm{p}$ values with multiple comparison testing (because of the increased likelihood of type I statistical error), we feel that the comparisons in table 4 are instructive in that they offer useful guidance in the management of contact lens wearers.

\section{Corneal cultures}

Twenty five corneal scrapes were performed on the keratitis patients surveyed in this study; the culture results are presented in table 5. A clinical decision was taken to execute a corneal scrape in two of 80 cases which were classified by the scoring system to be non-severe keratitis, and bacterial growth was detected in both cases. A corneal scrape was performed in 23 of the 38 cases classified as severe keratitis, yielding a positive culture result for bacteria in nine cases and fungus in one case.

\section{DISCUSSION}

In the epidemiological studies of Poggio et $a l^{17}$ and Cheng et $a l,{ }^{18}$ participating clinicians were required to include cases of microbial keratitis based on the following clinical criterion: “...a corneal stromal infiltrate with an overlying epithelial abnormality (ulceration) clinically diagnosed as microbial keratitis ... [and] ... antimicrobial treatment ..." In essence, this criterion relates to clinically severe microbial keratitis, which is broadly equivalent to the category of "severe keratitis" in the present study; as such, a comparison of results between these studies can be made.

The finding in this study of a higher overall incidence of severe keratitis in wearers who sleep in contact lenses compared with those who only use lenses during waking hours is consistent with previous reports. ${ }^{17-19}$ However, the annual incidence of severe keratitis per 10000 wearers with hydrogel lenses worn on a daily wear basis reported here $(6.4$ (95\% CI 4.1 to 9.9$)$ ) is about $1.7 \times$ greater than the figures of Poggio et $a^{17}$ (4.1 (95\% CI 2.9 to 5.2)) and Cheng et al ${ }^{18}$ (3.5 (95\% CI 2.7 to 4.5$)$ ). Furthermore, we report a much higher annual incidence (about $4.7 \times$ ) of severe keratitis for hydrogel lenses worn on an extended wear basis (96.4 (95\% CI 37.5 to 245.2)) relative to the figures reported by Poggio et al ${ }^{17}$ (20.9 (95\% CI 15.1 to 26.7$)$ ) and Cheng et al ${ }^{18}$ (20.0 (95\% CI 10.3 to 35.0)).

There are various possible reasons for the higher incidence figures relating to severe keratitis reported in this study. The highly managed, single centre methodology adopted here is likely to have resulted in a higher rate of capture of severe keratitis cases compared with the multicentre approach of Poggio et $a l^{17}$ and Cheng et al ${ }_{18}^{18}$ whereby reliance was placed upon on full compliance in terms of case capture and reporting by 612 and 379 doctors, respectively, spread over large geographic areas. A higher capture rate will result in higher and necessarily more accurate incidence figures. Another reason for the above discrepancy relates to the choice of criterion adopted in relation to the classification of

Table 3 Annual incidence and relative risk of non-severe and severe keratitis in contact lens wear

\begin{tabular}{|c|c|c|c|c|c|}
\hline \multirow[b]{2}{*}{ Wearing modality } & \multirow[b]{2}{*}{ Lens type } & \multicolumn{2}{|l|}{ Annual incidence* } & \multicolumn{2}{|l|}{ Relative risk $\ddagger$} \\
\hline & & Non-severe keratitis & Severe keratitis & Non-severe keratitis & Severe keratitis \\
\hline Daily wear & $\begin{array}{l}\text { Rigid } \\
\text { Hydrogel daily disposable } \\
\text { Hydrogel } \\
\text { Silicone hydrogel }\end{array}$ & $\begin{array}{l}5.7(2.2 \text { to } 14.7) \dagger \\
9.1(5.5 \text { to } 15.1) \\
14.1(10.4 \text { to } 19.0) \\
55.9(9.9 \text { to } 309.7)\end{array}$ & $\begin{array}{l}2.9(0.8 \text { to } 10.4) \\
4.9(2.5 \text { to } 9.6) \\
6.4(4.1 \text { to } 9.9) \\
0.0(0.0 \text { to } 210.1)\end{array}$ & $\begin{array}{l}0.4(0.2 \text { to } 1.1) \\
0.7(0.4 \text { to } 1.2) \\
1.0 \\
4.0(0.6 \text { to } 28.7)\end{array}$ & $\begin{array}{l}0.5(0.1 \text { to } 1.9) \\
0.8(0.3 \text { to } 1.8) \\
1.0 \\
\S\end{array}$ \\
\hline Extended wear & $\begin{array}{l}\text { Rigid } \\
\text { Hydrogel } \\
\text { Silicone hydrogel }\end{array}$ & $\begin{array}{l}0.0(0.0 \text { to } 1758.8) \\
48.2(13.2 \text { to } 174.0) \\
98.8(60.0 \text { to } 162.5)\end{array}$ & $\begin{array}{l}0.0(0.0 \text { to } 1758.8) \\
96.4(37.5 \text { to } 245.2) \\
19.8(6.7 \text { to } 58.0)\end{array}$ & $\begin{array}{l}\S \\
3.4(0.8 \text { to } 14.1) \\
7.0(3.9 \text { to } 12.7)\end{array}$ & $\begin{array}{l}\S \\
15.2(5.2 \text { to } 44.4) \\
3.1(0.9 \text { to } 10.5)\end{array}$ \\
\hline
\end{tabular}

*Number of cases per 10,000 wearers per year.

†9\% confidence limits.

†Calculated independently for non-severe and severe keratitis, taking "daily wear hydrogel" as the referent in each case.

§Indeterminable because of an annual incidence of zero. 
Table 4 Statistical test results of comparisons of incidences of non-severe and severe keratitis

\begin{tabular}{|c|c|c|c|c|c|c|c|c|c|c|c|}
\hline \multirow{2}{*}{$\begin{array}{l}\text { Modality of wear } \\
\text { Lens type }\end{array}$} & \multirow[b]{2}{*}{ Type of keratitis } & \multicolumn{2}{|c|}{$\begin{array}{l}\text { Daily wear } \\
\text { Hydrogel daily } \\
\text { disposable }\end{array}$} & \multicolumn{2}{|c|}{$\begin{array}{l}\text { Daily wear } \\
\text { Hydrogel }\end{array}$} & \multicolumn{2}{|c|}{$\begin{array}{l}\text { Daily wear } \\
\text { Silicone hydrogel }\end{array}$} & \multicolumn{2}{|c|}{$\begin{array}{l}\text { Extended wear } \\
\text { Hydrogel }\end{array}$} & \multicolumn{2}{|c|}{$\begin{array}{l}\text { Extended wear } \\
\text { Silicone hydrogel }\end{array}$} \\
\hline & & $\overline{\chi^{2}}$ & p Value & $\chi^{2}$ & p Value & $\overline{\chi^{2}}$ & p Value & $\overline{\chi^{2}}$ & p Value & $\overline{\chi^{2}}$ & p Value \\
\hline $\begin{array}{l}\text { Daily wear } \\
\text { Rigid } \\
\text { Daily wear } \\
\text { Hydrogel daily disposable } \\
\text { Daily wear } \\
\text { Hydrogel } \\
\text { Daily wear } \\
\text { Silicone hydrogel } \\
\text { Extended wear Hydrogel }\end{array}$ & $\begin{array}{l}\text { Non-severe } \\
\text { Severe } \\
\text { Non-severe } \\
\text { Severe } \\
\text { Non-severe } \\
\text { Severe } \\
\text { Non-severe } \\
\text { Severe } \\
\text { Non-severe } \\
\text { Severe }\end{array}$ & $\begin{array}{l}0.4 \\
0.5\end{array}$ & $\begin{array}{l}0.46 \\
0.73\end{array}$ & $\begin{array}{l}3.2 \\
1.2 \\
2.1 \\
0.4\end{array}$ & $\begin{array}{l}0.09 \\
0.40 \\
0.15^{\star} \\
0.53^{*}\end{array}$ & $\begin{array}{l}6.3 \\
\dagger \\
4.0 \\
\dagger \\
2.2 \\
\dagger\end{array}$ & $\begin{array}{l}0.12 \\
\dagger \\
0.16 \\
\dagger \\
0.23 \\
t\end{array}$ & $\begin{array}{l}8.7 \\
42.4 \\
6.1 \\
47.6 \\
3.3 \\
43.7 \\
0.0 \\
\dagger\end{array}$ & $\begin{array}{l}0.04 \\
0.0001 \\
0.06 \\
0.0002 \\
0.12 \\
0.0003 \\
1.00 \\
\dagger\end{array}$ & $\begin{array}{l}48.6 \\
6.1 \\
67.0 \\
5.0 \\
57.3 \\
3.7 \\
0.3 \\
+ \\
1.0 \\
5.3\end{array}$ & $\begin{array}{l}0.0001 \text { * } \\
0.04 \\
0.0001 \text { * } \\
0.06 \\
0.0001 \text { * } \\
0.09 \\
1.00 \\
+ \\
0.55 \\
0.04\end{array}$ \\
\hline
\end{tabular}

corneal infiltrative events as representing non-severe versus severe keratitis. The sensitivity of the assessment criterion in making this differential diagnosis can be adduced from inspection of figure 2, and is demonstrated with respect to the scoring system used in this study in table 6. As can be seen from this table, when the threshold increases, the incidence of non-severe keratitis increases and the incidence of severe keratitis decreases. In addition, the employment of a clinical severity matrix such as that used in the present work has the advantage of greatly reducing between observer variability in differentially diagnosing non-severe from severe keratitis.

The relative risk of developing severe keratitis for extended wear versus daily wear of hydrogel lenses was higher in this study $(15.2 \times)$ compared with the data of Schein et al ${ }^{20}(5.2 \times)$ and Cheng et $a l^{18}(5.7 \times)$. It is interesting to observe that, in the present study, relative risk is not markedly affected by the choice of threshold clinical severity score; for example, adopting threshold scores of 6,8 , and 10 gives rise to relative risk values for severe keratitis for extended wear relative to daily wear of hydrogel lenses of $10.9 \times, 15.2 \times$, and $16.0 \times$, respectively (see table 6).
Stapleton $e t a^{21}$ reported that the risk of sterile keratitis (which equates to "non-severe keratitis" in the present study) for extended wear hydrogel lenses, relative to daily wear hydrogel lenses, was $1.2 \times$, whereas we have found a relative risk of $3.4 \times$ (although our value was not statistically significantly different from unity). The reasons for the discrepancies in relative risk reported in this study compared with the data of Schein et al, ${ }^{20}$ Cheng et al, ${ }^{18}$ and Stapleton et $a l^{21}$ are unclear, but could be because of differences in assumptions relating to the proportion of wearers in the population using lenses on a daily versus extended wear basis.

A key finding of this study is the five times decreased risk of severe keratitis with silicone hydrogel lenses compared to that with hydrogel lenses when worn on an extended wear basis. The essential distinction between these lens types is the superior oxygen transmissibility of silicone hydrogel lenses. ${ }^{4}$ Our observation lends support to the notion that the pathogenesis of severe keratitis is significantly related to corneal oxygen availability. Solomon et al ${ }^{22}$ confirmed such an association in a rabbit eye model by demonstrating higher rates of severe microbial keratitis in animals subjected to

Table 5 Outcomes of cultures of corneal scrapes

\begin{tabular}{|c|c|c|c|c|c|c|}
\hline Patient code & Age & Sex & Wearing modality & Lens type & $\begin{array}{l}\text { Clinical severity } \\
\text { score }\end{array}$ & Culture outcome \\
\hline 82 & 26 & $\mathrm{~F}$ & Daily wear & Hydrogel & 7 & Staphylococcus spp* \\
\hline 48 & 30 & $\mathrm{~F}$ & Extended wear & Silicone hydrogel & 8 & Bacillus spp \\
\hline 36 & 32 & $M$ & Extended wear & Hydrogel daily disposable & 9 & No growth 5 days \\
\hline 58 & 40 & $M$ & Daily wear & Hydrogel & 9 & No growth 5 days \\
\hline 197 & 23 & $M$ & Daily wear & Hydrogel & 9 & No growth 5 days \\
\hline 205 & 34 & $M$ & Daily wear & Hydrogel & 9 & No growth 5 days \\
\hline 209 & 35 & $\mathrm{~F}$ & Extended wear & Hydrogel & 9 & No growth 5 days \\
\hline 362 & 83 & $\mathrm{~F}$ & Daily wear & Rigid & 9 & Stenotrophomonas maltophilia \\
\hline 364 & 23 & $\mathrm{~F}$ & Daily wear & Hydrogel daily disposable & 9 & No growth 5 days \\
\hline 44 & 61 & $M$ & Extended wear & Silicone hydrogel & 10 & Propionibacterium acnes \\
\hline 185 & 20 & $\mathrm{~F}$ & Daily wear & Hydrogel & 10 & Mixed coliforms \\
\hline 206 & 51 & $\mathrm{~F}$ & Daily wear & Hydrogel daily disposable & 10 & Candida albicans \\
\hline 369 & 30 & $\mathrm{~F}$ & Daily wear & Hydrogel & 10 & Acinetobacter spp \\
\hline 92 & 24 & $\mathrm{~F}$ & Daily wear & Hydrogel & 11 & No growth 5 days \\
\hline 95 & 20 & $\mathrm{~F}$ & Extended wear & Hydrogel & 11 & Staphylococcus spp* \\
\hline 195 & 31 & $M$ & Daily wear & Rigid & 11 & No growth 5 days \\
\hline 291 & 60 & $\mathrm{~F}$ & Extended wear & Silicone hydrogel & 11 & No growth 5 days \\
\hline 41 & 27 & $\mathrm{~F}$ & Daily wear & Hydrogel daily disposable & 12 & No growth 5 days \\
\hline 213 & 48 & $M$ & Daily wear & Hydrogel & 12 & Mixed coliforms \\
\hline 295 & 24 & $\mathrm{~F}$ & Daily wear & Hydrogel daily disposable & 12 & No growth 5 days \\
\hline 365 & 19 & $M$ & Daily wear & Hydrogel & 12 & No growth 5 days \\
\hline 372 & 21 & $M$ & Daily wear & Hydrogel & 13 & No growth 5 days \\
\hline 375 & 47 & $M$ & Daily wear & Hydrogel & 14 & Pseudomonas spp \\
\hline 93 & 29 & $M$ & Extended wear & Hydrogel & 16 & Pseudomonas spp \\
\hline 273 & 25 & $M$ & Extended wear & Silicone hydrogel & 19 & Pseudomonas spp \\
\hline
\end{tabular}




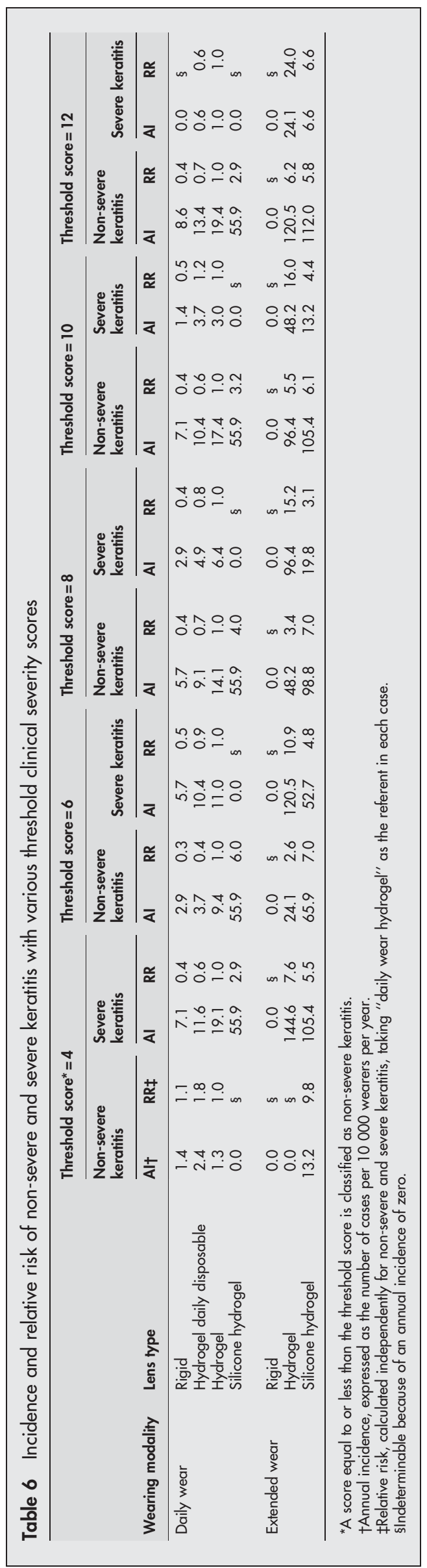

lower levels of corneal oxygenation. Ren et $\mathrm{l}^{23}$ demonstrated that Pseudomonas bacteria are less likely to attach to corneal epithelial cells in the rabbit cornea (bacterial attachment to the cornea being a necessary precursor for infection) when contact lenses of higher oxygen transmissibility are worn.

The above discussion has concentrated on our findings with respect to silicone hydrogel lenses. Daily disposable lenses are another recent innovation in the contact lens field. Notwithstanding the advantages of this lens type in terms of wearer convenience and avoiding adverse effects related to long term deposit formation, ${ }^{12}$ we have found no statistically significant difference in the incidences of severe keratitis among wearers of daily wear daily disposable lenses versus daily wear hydrogel lenses that are replaced less frequently.

Of the 23 corneal scrapes performed on patients deemed to have severe keratitis (according to the scoring system used), 10 were culture positive. This is equivalent to a culture positive rate of $43 \%$, which is consistent with that observed in other contact lens studies. ${ }^{18}{ }^{20}$ Pseudomonas species, which are generally considered to be the most virulent bacterial pathogen in contact lens related keratitis, ${ }^{24}$ were associated with the three highest clinical severity scores of the patients on whom a corneal scrape was performed.

We conclude that (a) a clinical severity matrix has considerable utility in assessing contact lens related keratitis, (b) there is a significantly higher incidence of severe keratitis in wearers who sleep in contact lenses compared with those who only use lenses during the waking hours and (c) those who choose to sleep in lenses should be advised to wear silicone hydrogel lenses as they carry a five times lower risk of severe keratitis for extended wear compared with hydrogel lenses.

\section{ACKNOWLEDGEMENTS}

The authors thank colleagues who work in the acute service of the Royal Eye Hospital, Manchester-in particular John Uddin and Linda Raynor-for their enthusiastic and conscientious participation in this survey. The authors also wish to thank Julie Morris for her valuable statistical advice, and to acknowledge the excellent service provided by the Microbiology Department of the Central Manchester and Manchester Children's University Hospitals National Health Service Trust and the advice of Malcolm Armstrong.

\section{Authors' affiliations}

P B Morgan, N Efron, E A Hill, Eurolens Research, Department of Optometry, The University of Manchester, UK

M K Raynor, M A Whiting, A B Tullo, Royal Eye Hospital, Manchester, UK

\section{REFERENCES}

1 Sankaridurg PR, Sweeney DF, Holden BA, et al. Comparison of adverse events with daily disposable hydrogels and spectacle wear: results from a 12 month prospective clinical trial. Ophthalmology 2003;1 10:2327-34.

2 Solomon OD, Freeman MI, Boshnick EL, et al. A 3-year prospective study of the clinical performance of daily disposable contact lenses compared with frequent replacement and conventional daily wear contact lenses. Contact Lens Assoc Ophthalmol J 1996;22:250-7.

3 Sweeney DF. Silicone hydrogels. Continuous wear contact lenses. 2nd ed. Oxford: Butterworth-Heinemann, 2004.

4 Alvord L, Court J, Davis T, et al. Oxygen permeability of a new type of high Dk soft contact lens material. Optom Vis Sci 1998;75:30-6.

5 Hingorani $M$, Christie C, Buckley RJ. Ulcerative keratitis in a person wearing daily disposable contact lenses. Br J Ophthalmol 1995;79:1138.

6 Lim L, Loughnan MS, Sullivan $\sqcup$. Microbial keratitis associated with extended wear of silicone hydrogel contact lenses. Br J Ophthalmol 2002;86:355-7.

7 Whiting MA, Raynor MK, Morgan PB, et al. Continuous wear silicone hydrogel contact lenses and microbial keratitis. Eye 2004;18:935-7.

8 Aasuri MK, Venkata N, Kumar VM. Differential diagnosis of microbial keratitis and contact lens-induced peripheral ulcer. Eye Contact Lens 2003;29(1 Suppl):S60-2.

9 Sweeney DF, Jalbert I, Covey M, et al. Clinical characterization of corneal infiltrative events observed with soft contact lens wear. Cornea 2003;22:435-42.

10 Baum J, Donshik PC. Corneal infiltrates associated with soft contact lens wear (Letter). Cornea 2003;22:435-42.

11 Senn SJ, Samson WB. Estimating hospital catchment populations. Statistician 1982;31:81-96. 
12 Morgan PB, Efron N. Trends in UK contact lens prescribing 2003. Optician 2003;225(5904):34-5.

13 Morgan PB. Taking the pulse of the UK contact lens market. Optician 2004;227(5954):26-7.

14 www.statistics.gov.uk/cci/nugget.asp?id =6, accessed 4 May 2004

15 Wilson EB. Probable inference, the law of succession, and statistical inference. J Am Stat Assoc 1927;22:209-12.

16 Bland MJ, Altman DG. The odds ratio. BMJ 2000;320:1468.

17 Poggio EC, Glynn RJ, Schein OD, et al. The incidence of ulcerative keratitis among users of daily-wear and extended-wear soft contact lenses. N Engl J Med 1989;321:779-83.

18 Cheng KH, Leung SL, Hoekman HW, et al. Incidence of contact-lensassociated microbial keratitis and its related morbidity. Lancet 1999:354:181-5.

19 Schein OD, Buehler PO, Stamler JF, et al. The impact of overnight wear on the risk of contact lens-associated ulcerative keratitis. Arch Ophthalmol 1994;112:186-90.
20 Schein OD, Glynn RJ, Poggio EC, et al. The relative risk of ulcerative keratitis among users of daily-wear and extended-wear soft contact lenses. A casecontrol study. Microbial Keratitis Study Group. N Engl J Med 1989:321:773-8.

21 Stapleton F, Dart J, Minassian D. Nonulcerative complications of contact lens wear. Relative risks for different lens types. Arch Ophthalmol 1992;110:1601-6.

22 Solomon OD, Loff H, Perla B, et al. Testing hypotheses for risk factors for contact lens-associated infectious keratitis in an animal model. Contact Lens Assoc Ophthalmol J 1994:20:109-13.

23 Ren DH, Petroll WM, Jester JV, et al. The relationship between contact lens oxygen permeability and binding of Pseudomonas aeruginosa to human corneal epithelial cells after overnight and extended wear. Contact Lens Assoc Ophthalmol J 1999:25:80-100.

24 Fleiszig SM, Evans DJ. The pathogenesis of bacterial keratitis: studies with Pseudomonas aeruginosa. Clin Exp Optom 2002;85:271-8.

Familial keratoconus maps to chromosome 2p24

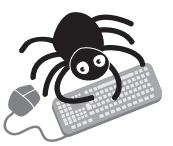

Please visit the British Journal of

Ophthalmology website [www. bjophthalmol. com] for a link to the full text of this article.
D esearchers have taken an important step to understanding keratoconus (KC), a common reason for corneal transplantation in the developed world. They did so with the first scan of the whole genome in different races, identifying a new locus likely to be responsible for a significant proportion of cases of isolated familial KC.

Genotyping followed by linkage analysis in a small sample of families suggested linkage on chromosome $2 \mathrm{p}$ for marker D2S305 but not to chromosomes 16q, 18p, or 21q, previously suggested for familial KC. Typing the full complement of 28 families for 36 other markers across an $80 \mathrm{cM}$ region spanning D2S319 and D2S286 suggested linkage at D2S305. Further analysis suggested significant linkage to 2p24. Marker alleles segregated at 2p24 in affected members in 17 of the 28 families, and the locus for $\mathrm{KC}$ was placed in a $1.69 \mathrm{Mb}$ region, within which were two intervals common to all 17. None of the five known genes in the two intervals yet has a known role in human disease, but research is continuing into the expression of all candidate genes

The families were of Caucasian, Arabic, or Caribbean African descent. Among the 253 family members at least two per family had the disorder. The cause of $\mathrm{KC}$ is still unknown. The familial form accounts for $6-10 \%$ of cases. Mostly it seems to show autosomal dominant inheritance, though autosomal recessive and, rarely, X linked inheritance has been claimed. Various other loci have been mooted-in restricted, homogeneous populations-maybe pointing to genetic heterogeneity.

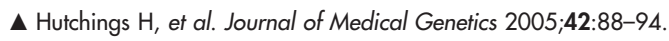

